\title{
Energy Efficient Target Tracking Scheme for Wireless Sensor Networks
}

\author{
Ashvini Pawar' ${ }^{1}$, S. A. Nagtilak ${ }^{2}$ \\ ${ }^{1}$ Department of Information Technology Sinhgad Technical Education Society’s SKNCOE, Pune, India \\ ${ }^{2}$ Professor, Department of Information Technology, Sinhgad Technical Education Society’s SKNCOE, Pune, India
}

\begin{abstract}
Target tracking in WSN has a lot of applications in military fields and surveillance purpose. Target tracking in WSN is more challenging because WSNs have issues such as limited battery power, unpredictable environments, high mobility of nodes as well as targets and failure of sensor nodes at runtime etc. This paper proposes a new polygon based target-tracking scheme, which is a predictive, and cluster based scheme. In this scheme, the clusters are polygon shaped, so the whole network is arranged in the form of polygons. The sensor nodes are interconnected with their neighboring nodes to form the edges of the polygons. Proposed scheme is more energy efficient than the existing schemes because only selected nodes of the polygon are kept active during the tracking process rather than keeping all the nodes active. Edge detection algorithm is used to send the message to the sensors of the next polygon and activate them before target's arrival in that polygon. A-star algorithm is used to choose the optimal nodes and to find the optimal routing path from source node to the sink. This routing scheme improves the energy efficiency as well as tracking accuracy of WSN by forwarding the target-tracking message via the optimal shortest path.
\end{abstract}

Keywords: A-star, Edge detection, Energy efficiency, Sensor selection, Target tracking, Wireless sensor network

\section{Introduction}

Recent researches in wireless communication, digital electronics and microelectronics have enabled the deployment of large-scale sensor networks over a vast remote area. Wireless Sensor Networks are deployed using a large number of sensor nodes, which can gather the data from the remote environment and process that data to generate the useful information. These sensors are of different types such as thermal, infrared, acoustic etc., which are used to monitor different environment conditions like temperature, light and noise respectively. Wireless sensor networks have a lot of applications like surveillance and communication in military areas; monitoring the conditions of crops in agriculture lands; diagnostic of diseases in hospitals etc.

Target tracking [2] is one of the most important applications of WSN but its challenging also because of the constraints in WSN such as a limited battery of sensor nodes, unpredictable environments, node failure at run time and high mobility of the target. Existing target tracking schemes are mainly clustering based or tree based. In tree-based schemes [9], the sensor nodes are arranged in the form of a tree structure and the root of the tree is chosen dynamically. This root node sends information to the sink. The Clustering based target tracking is mainly of two types: static clustering and dynamic clustering. In static clustering [3], the cluster heads are assigned to the respective sensor nodes at the time of network formation and they cannot changed later. Dynamic clustering schemes [3] are categorized into the prediction based [4] and non- prediction based. In the prediction-based scheme, when target moves from one cluster head to the other, the current cluster head makes prediction about targets movement and wakeup a cluster head of next cluster. In non- prediction based schemes, the energy saving is not an issue but the proper sensor selection has given the more importance.
This paper proposes a polygon based target-tracking framework [14] using edge detection and A-star algorithm. This method is a prediction based, and the network is arranged in the form of polygons.

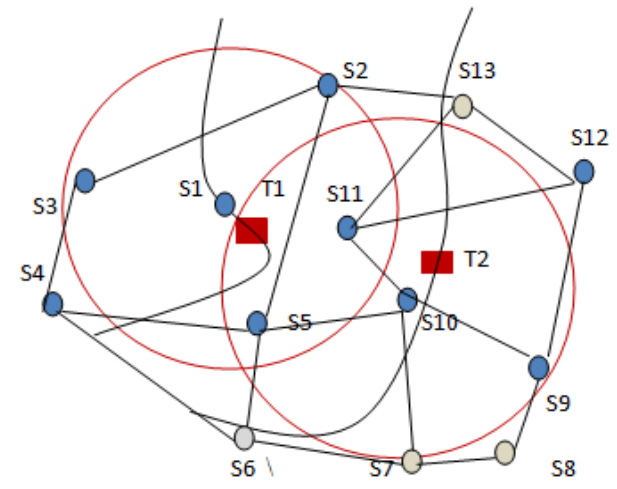

Figure 1: Target tracking in WSN

As shown in the Figure 1, when the target crosses the edge between two polygons, the edge detection algorithm is invoked and the sensors of that polygon are activated. The sensors, which are active, are shown by blue color and the sensors, which are in the sleep mode, are shown by gray color. As shown in above Figure 1, the polygon tracking method using edge detection and A-star algorithm is able to track the multiple targets at a time. This method is more energy efficient and accurate than existing target tracking methods.

\section{Related Work}

In paper [5], author introduced sensor-scheduling method to minimize the tradeoff between tracking performance and energy consumption. This method uses bottom up approach, in which different sensing, motion and cost models are 


\section{International Journal of Science and Research (IJSR) \\ ISSN (Online): 2319-7064 \\ Index Copernicus Value (2013): 6.14 | Impact Factor (2014): 5.611}

considered with increasing levels of difficulty. At first level, target is only observed within the sensing range of an active sensor i.e. sensing regions of different sensors do not overlap. At next level, sensing ranges of sensors are overlapping. Finally, the scenario is considered in which target locations and sensor's observations assume values on continuous spaces. However, this method is applicable only when central controller is present in the network.

In paper [6], author introduced a scheme PPSS (Probability based prediction and sleep scheduling protocol) to improve the energy efficiency of proactive wake-up. PPSS not only predicts targets next location, but also describes probabilities with which it moves in the all directions. Based on prediction results, PPSS enhances the energy efficiency by reducing a number of proactively awaken nodes and controlling their active time. PPSS does not use optimization techniques i.e. it does not impose performance constraints when reducing the energy consumption. Prediction method of PPSS cannot cover special cases such as target movement with abrupt direction changes.

In paper [7], author proposed GNS scheme (Global Node Selection) for target tracking. GNS scheme requires knowledge of all the neighbors of each sensor nodes for target tracking. GNS scheme does not allow the node failure scenarios.

In paper [8], author proposed ANS scheme (Autonomous Node Selection). This scheme is modification to GNS as it uses distributed strategy. It requires only local node information. ANS is more desirable than GNS. ANS does not require whole network to be reconstructed when nodes will be added or removed.

In paper [9], author proposed a tree-based approach for target tracking called Dynamic Convoy Tree Based Collaboration (DCTC). Convoy tree is dynamically constructed, and when the target enters the detection region, sensor nodes collaborate with each other and select a root. Root node collects all information about the target and sends to the user. In DCTC technique, when the target moves far from the root, more energy is consumed in the communication between the target and the root node.

Table 1: Literature Survey

\begin{tabular}{|c|c|c|c|}
\hline Sr.no. & Existing Method & Advantages & Disadvantages \\
\hline 1. & Sensor Scheduling & $\begin{array}{c}\begin{array}{c}\text { This method minimizes the tradeoff between } \\
\text { tracking performance and energy } \\
\text { consumption. }\end{array} \\
\end{array}$ & $\begin{array}{c}\text { This method always requires a central } \\
\text { controller. }\end{array}$ \\
\hline 2. & $\begin{array}{c}\text { Probability-Based Prediction and Sleep } \\
\text { Scheduling (PPSS) }\end{array}$ & $\begin{array}{c}\begin{array}{c}\text { This method improves the efficiency of } \\
\text { proactive woke up by applying prediction } \\
\text { scheme. }\end{array} \\
\end{array}$ & $\begin{array}{l}\text { This method imposes no performance } \\
\text { constraints when reducing energy } \\
\text { consumption. }\end{array}$ \\
\hline 3. & Global Node Selection Algorithm & This method is simple to implement. & $\begin{array}{c}\text { The assumption that each node must know } \\
\text { the location of all nodes can become } \\
\text { overhead when network size grows. }\end{array}$ \\
\hline 4. & Autonomous Nod & $\begin{array}{l}\text { 1) In the case of the large network, memory } \\
\text { requirements are not burdensome as that of } \\
\text { GNS. } \\
\text { 2) ANS is more desirable than GNS because } \\
\text { the whole network does not need to } \\
\text { reconfigure after addition or removal of extra } \\
\text { nodes. }\end{array}$ & $\begin{array}{l}\text { It cannot handle the scenario in which } \\
\text { multiple targets are in detection range if the } \\
\text { same nodes i.e. multi- target issues. }\end{array}$ \\
\hline 5. & $\begin{array}{l}\text { Dynamic Convoy Tree Based } \\
\text { Collaboration }\end{array}$ & $\begin{array}{l}\text { For power saving, most of the sensor nodes } \\
\text { stay asleep before target arrives. }\end{array}$ & $\begin{array}{l}\text { The energy consumption increases with } \\
\text { increase of the velocity of the target. } \\
\text { Reconfiguration of the tree structure } \\
\text { requires more energy. }\end{array}$ \\
\hline 6. & $\begin{array}{ll}\text { 1) } & \begin{array}{l}\text { Distributed Election Winner } \\
\text { Algorithm (DENA) }\end{array} \\
\text { 2) } & \begin{array}{l}\text { Intensity Based Localization } \\
\text { Algorithm (ILA) }\end{array}\end{array}$ & $\begin{array}{l}\text { Location of an event can be calculated in a } \\
\text { distributed strategy } \\
\text { without the need to gather any information } \\
\text { on a destination node. }\end{array}$ & $\begin{array}{l}\text { 1) ILA needs information of all sink nodes } \\
\text { instead of considering subset of them. } \\
\text { 2) DENA used DDB that causes additional } \\
\text { delay. }\end{array}$ \\
\hline 7. & $\begin{array}{l}\text { 1) Kalman filter } \\
\text { 2) Machine learning algorithms }\end{array}$ & $\begin{array}{l}\text { Provides accurate target tracking and it is } \\
\text { robust in any case of a noisy data. }\end{array}$ & $\begin{array}{l}\text { No special technique is provided for } \\
\text { reducing energy consumption. }\end{array}$ \\
\hline 8. & Distributed processing of RSSI & $\begin{array}{c}\text { Minimizes power consumption, and reduces } \\
\text { latency at the central base station. }\end{array}$ & $\begin{array}{l}\text { False detection of target occurs in some } \\
\text { cases. }\end{array}$ \\
\hline 9. & $\begin{array}{l}\text { 1) BSS algorithm } \\
\text { 2) Clustering and selecting algorithm }\end{array}$ & $\begin{array}{l}\text { This method can track multiple targets within } \\
\text { the sensing range of same active sensor. }\end{array}$ & $\begin{array}{c}\text { Energy consumption is more for tracking } \\
\text { multiple targets. }\end{array}$ \\
\hline
\end{tabular}

In paper [10], author introduced intensity Based Event Localization Scheme. In this method, two algorithms are introduced for event detection and localization, distributed election winner algorithm (DENA) and intensity based localization algorithm (ILA). DENA decides which sensor node is closer to the target event (i.e. winner node) and sends a notification message to all sensor nodes about the winner node. ILA calculates the position of the event and this takes place at the winner node determined by DENA. In this method, location estimation occurs in a distributed manner, so there is no need to gather any tracking information on the sink node. 


\section{International Journal of Science and Research (IJSR) \\ ISSN (Online): 2319-7064 \\ Index Copernicus Value (2013): 6.14 | Impact Factor (2014): 5.611}

In paper [11], author introduced a new method for target tracking which combines machine learning with Kalman filter to calculate the positions of the moving target. Radio fingerprints of RSSI (Received signal strength index) are first collected from the target movement and then the collected database is used with machine learning algorithms. This method provides accurate tracking, and it is observed to be robust in any case of noisy data. However, this method does not provide any technique to reduce the energy consumption while target tracking.

In paper [12], author proposed RSSI scheme for target movement detection using the signal strength of sensor nodes. In WSN, sensor nodes continuously communicate with each other by exchanging the messages. When the target arrives into the network, signal strength of sensor nodes reduces and nodes come to know that target has entered in the network. But this scheme has a drawback which is misinterpretation of signal strength reduction. Sometimes, sensor signal strength is purposely reduced for minimizing transmission power, but some nodes misinterpret this reduction in signal strength and generate false notification of target detection.

In paper [13], author proposed a new method for tracking non-cooperative multiple targets. This method uses both temporal and spatial information available for the tracking. Signals received by sensors are generally mixtures of signals from individual targets. For example, a sensor in a field of interest may receive signals from more than one target. First, using BSS (Blind Source Separation) algorithm, it separates the individual signals from received aggregate signals. Aggregate signals contain the signals from multiple indistinguishable targets and the noise. Then, through the analysis of the temporal and spatial correlation of the individual target signals, tracking algorithm determines the location of the target and its moving path. Clustering and selecting algorithms are used to remove noise from the aggregate signal. This method can track the multiple noncooperative targets accurately and precisely.

\section{Proposed Work}

Most of the existing target-tracking schemes are more energy consuming because they keep large number of unwanted sensor nodes active though most of them are not participating in the tracking process. Proposed scheme overcomes drawbacks of previous schemes and provides better-tracking accuracy.

In this scheme, only those nodes are kept active which are present in the active polygon and rest of the nodes are kept in sleep mode. Therefore, energy consumption is minimum. This method uses edge detection algorithm to construct further polygon in target's moving path and optimal selection algorithm to determine which sensor nodes should be kept active. Proposed scheme uses edge detection algorithm for detecting multiple targets at the same time.

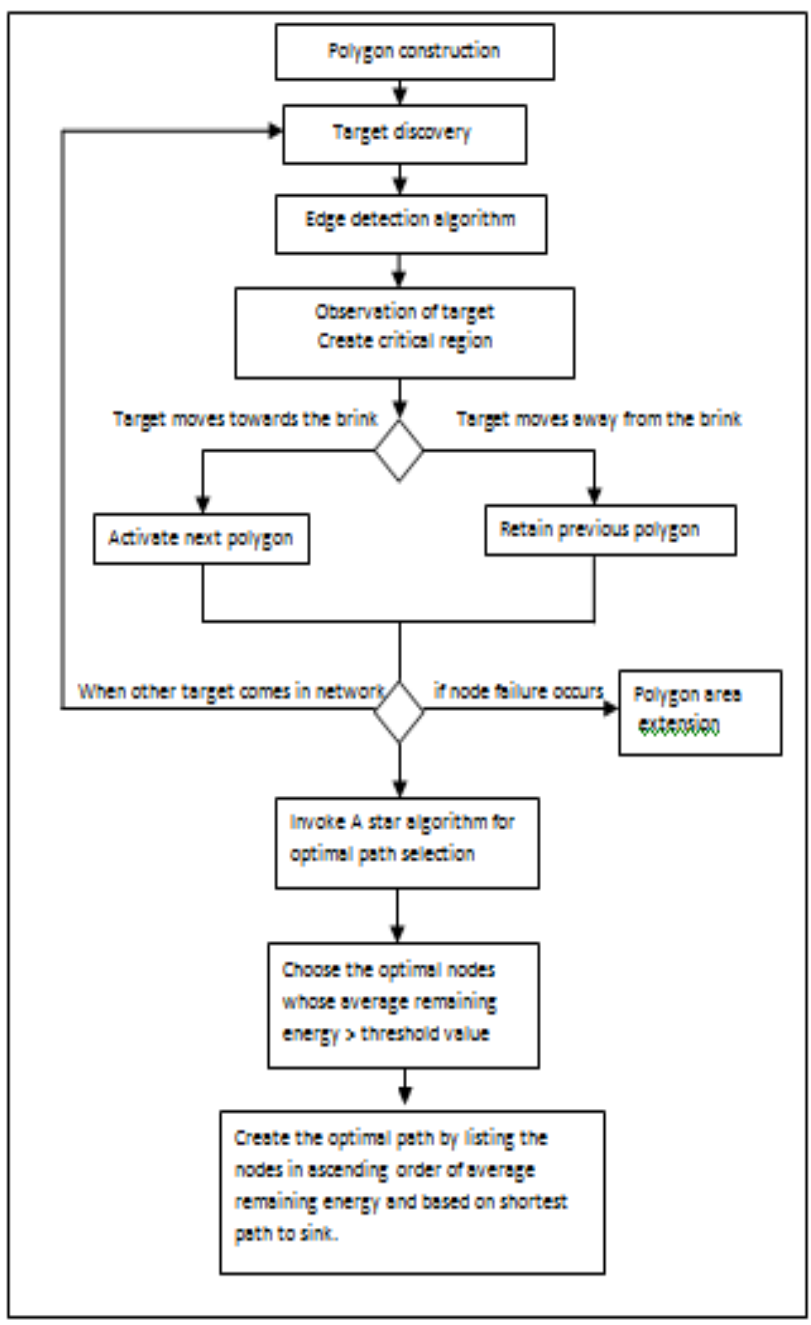

Figure 2: Proposed system framework

Figure 2 shows the proposed system framework. It is polygon based target tracking framework [1] with edge detection and A-star algorithm. First, the sensor nodes are deployed in the network then the network is arranged in the form of polygons. Node has three states [15]: active (node participates in tracking), inactive (node is in sleep mode), awakening (node awakes for a short period). In first step, some sensor node detects the target. This node becomes source node. This node sends message to its neighboring nodes. After that, when the target crosses the edge between the polygons then the edge detection algorithm is invoked. The source node sends message to the sensor nodes of the next polygon. The polygon in which target is present, is called as the active polygon. All the nodes in this polygon are made active. If the target moves towards the next polygon then the sensors of next polygon are made active; else, if the target is steady in the same polygon, then the sensor nodes observe the movements of the target. When the other target arrives in the network then steps from target detection are performed again in the same sequence. If any node fails at the run time, then this system is able to continue the tracking process by expanding the polygon area. When any sensor node fails, then that sensor node is not able to track the target movement and the tracking error increases. The tracking error failure rate of this system is much less than the tracking error failure rate of existing target tracking systems. 


\section{International Journal of Science and Research (IJSR) \\ ISSN (Online): 2319-7064}

Index Copernicus Value (2013): 6.14 | Impact Factor (2014): 5.611

A star algorithm is used in the proposed system for choosing the optimal path between source to the sink. This algorithm chooses optimal nodes from the nodes of the active polygon, and it keeps only the optimal nodes active rather than keeping all nodes of the polygon active. A star algorithm collects the two parameters of each sensor node; first the shortest path to each node from the sink (MH (n)). Then it collects the average remaining energy value of each sensor node. Based on these two parameters, it calculates the evaluation function for each node i.e. $\mathrm{f}(\mathrm{n})$. The nodes, for which the value of evaluation function is more, are chosen as the optimal nodes. These optimal nodes form the optimal path from the source to the sink. In addition, tracking information is transferred to the sink node through this optimal path.

\section{Algorithm for the proposed scheme:}

Step 1: Consider a WSN $G=\left(V_{x} E\right)$ where V is set of nodes and $\mathrm{E}$ is set of edges.

Construct the polygons in the network using planarization.

Step 2: Target emits the signal and closest sensor node detects that signal.

Send notification to neighboring nodes and the sink.

Step 3: Identify the critical region and observe the target.

Step 4: If the target crosses the edge between the polygons, then invoke edge detection algorithm.

Send a message to the nodes of the future polygon, and their state is changed to the active state.

Nodes of previous polygons change their state to inactive.

Else, retain the previous polygon.

Step 5: When other target comes into the network, go to step 2.

Step 6: If any node failure occurs then expand polygon region.

Step 7: Invoke A-star algorithm to choose the optimal path i.e. more energy efficient path.

\section{Mathematical Model}

1) Identify the nodes

- Let $\mathrm{S}$ be the set of sensor nodes

- $\mathrm{S}=\{\mathrm{s} 1, \mathrm{~s} 2, \mathrm{~s} 3 \ldots\}$

2) Calculation for energy consumption

- Energy of the sensor node is calculated as: $\mathrm{e}(\mathrm{s} 1)=\mathrm{I}_{\mathrm{s} 1}-\mathrm{R}_{\mathrm{s} 1}$

- Where $\mathrm{I}_{\mathrm{s} 1}$ the initial energy applied to the sensor node. For this system, initial energy of sensor is assumed 0.5 Joule.

- $\mathrm{R}_{\mathrm{s} 1}$ is the remaining energy of the sensor node. The remaining energy of the sensor node is 0.25 Joule after the one execution of the tracking.

- Total energy consumption of the system is $\mathrm{Es}=\mathrm{T}^{*}(\mathrm{e}(\mathrm{s} 1)+\mathrm{e}(\mathrm{s} 2)+\mathrm{e}(\mathrm{s} 3) \ldots . \mathrm{e}(\mathrm{sn}))$

- Where e(s1),e(s2)...ee(sn) are the energy consumed by the active sensor nodes $\mathrm{s} 1, \mathrm{~s} 2, \mathrm{~s} 3$ etc. $\mathrm{n}$ is the number of active sensor nodes.

- Where, $\mathrm{T}$ is the time for execution of the algorithm. This time is obtained by the function in Java by calculating the time of the system at starting and at the end of the algorithm.
- Let $\mathrm{A}$ be the current time of the system, defined before the execution of the algorithm.

Let $\mathrm{B}$ be the current time of the system, defined before the execution of the algorithm. $\mathrm{T}=(\mathrm{B}-\mathrm{A}) / 1000$

3) Calculation of the Tracking Error Failure

- When the sensor node fails at runtime, the standard deviation in tracking signal is introduced, this causes tracking error.

- Let TEF be tracking error failure.

- $\mathrm{TEF}=\delta *$ (no. of nodes failed at runtime)

- $\mathrm{TEF}=\frac{\delta 8 \text { no.of nodes failed at runtime } * \mathrm{~T}}{\text { no.of active nodes }}$

- Where, $\delta$ is the standard deviation, which is approximately same for all nodes because sensors are homogeneous. So, $\delta=1.65$

$\mathrm{T}$ is the time required for the execution of the algorithm.

4) Calculation of the Tracking Ability Rate

- Tracking Ability Rate, i.e. TAR is the metric that can show the degree of successful tracking in a system against all the difficulties, such as the presence of high TEF.

- $\mathrm{TAR}=1 / \mathrm{TEFs}$

\section{Experiments}

Performance of the proposed algorithm is evaluated using the metrics such as energy consumption, tracking ability rate and tracking error failure. Experiment environment used here is a Windows 7 with $3.30 \mathrm{GHz}$ Core i3 CPU and 4GB Ram Memory.

\section{Results and Discussions}

\subsection{Energy Consumption}

Energy consumption for the system is calculated by considering the number of active sensor nodes. The total energy consumption of the system is the energy consumed by all the optimal nodes.

Table 2 shows the energy consumption values for the facetrack scheme and A-star scheme for target tracking in Joule.

Table 2: Energy Consumption for Face- Track and A-Star Scheme

\begin{tabular}{|c|c|c|}
\hline $\begin{array}{c}\text { No. of active } \\
\text { sensor nodes }\end{array}$ & $\begin{array}{c}\text { Energy Consumption for } \\
\text { Face-track scheme } \\
\text { (Existing System) } \\
\text { (in Joule) }\end{array}$ & $\begin{array}{c}\text { Energy Consumption } \\
\text { for A-star scheme } \\
\text { (Proposed system) } \\
\text { (in Joule) }\end{array}$ \\
\hline 3 & 0.3975 & 0.2625 \\
\hline 7 & 0.9275 & 0.6125 \\
\hline 11 & 1.4575 & 0.9625 \\
\hline 15 & 1.9875 & 1.3125 \\
\hline 19 & 2.5175 & 1.6625 \\
\hline 23 & 3.0475 & 2.0125 \\
\hline 27 & 3.5775 & 2.3625 \\
\hline
\end{tabular}

Figure 3 shows the comparison of energy consumption for target tracking using Face-track and energy consumption for tracking the multiple targets at a time using A-star algorithm. So, it can be seen that the energy consumption for tracking

\section{Volume 5 Issue 2, February 2016}




\section{International Journal of Science and Research (IJSR) \\ ISSN (Online): 2319-7064 \\ Index Copernicus Value (2013): 6.14 | Impact Factor (2014): 5.611}

the multiple targets using A-star scheme is much less than the Face track scheme.

Energy consumption (in Joule)

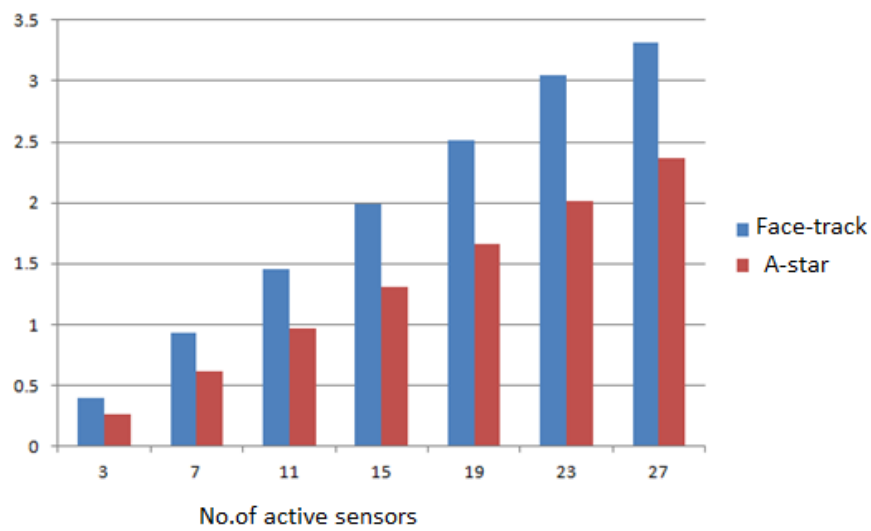

Figure 3: Energy consumption for Face-track and A-star

\subsection{Tracking Error Failure}

Some scenarios like node failure due to battery depletion can cause error in the tracking process at run time. This error is called tracking error failure.

Table 3: Tracking Error Failure for Face-Track and A- Star Scheme

\begin{tabular}{|c|c|c|}
\hline $\begin{array}{c}\text { No. of active } \\
\text { sensor nodes }\end{array}$ & $\begin{array}{c}\text { Tracking Error Failure for } \\
\text { Face-track scheme }\end{array}$ & $\begin{array}{c}\text { Tracking Error Failure } \\
\text { for A-star scheme }\end{array}$ \\
\hline 3 & 1.166 & 0.392 \\
\hline 7 & 0.499 & 0.168 \\
\hline 11 & 0.318 & 0.107 \\
\hline 15 & 0.232 & 0.078 \\
\hline
\end{tabular}

Above Table 3 shows the tracking error failure values for the face-track scheme and A-star scheme.

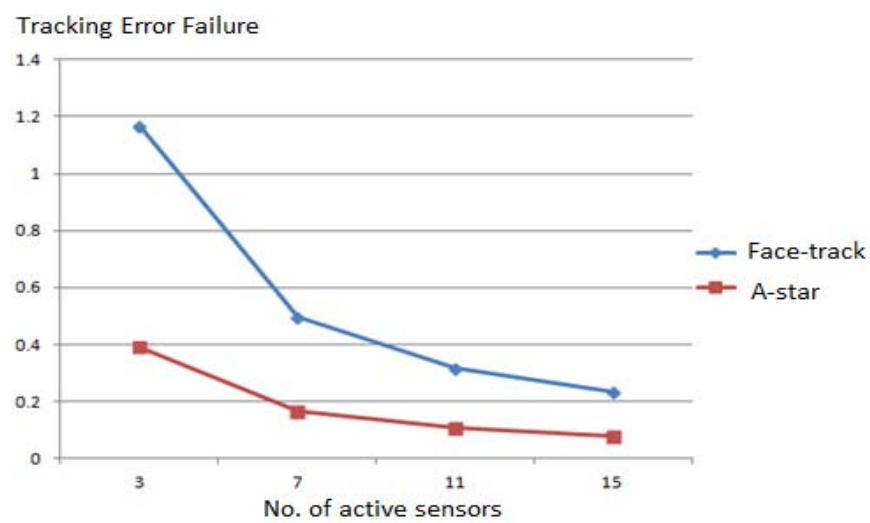

Figure 4: Tracking Error Failure for Face-track and A-star

Figure 4 shows the Tracking error failure of Face-track vs tracking error failure of A star. It can be seen that the Tracking error failure of the A-star is less as compared to the Face-track scheme. So, in case of node failure scenario, Astar scheme is more accurate tracking process than the facetrack scheme.

\subsection{Tracking Ability Rate}

Tracking Ability Rate is the accuracy of the tracking framework in presence of different faults in the network. For this system, the node failure is considered as the fault.
Table 4: Tracking Ability Rate for Face Track and A- Star Scheme

\begin{tabular}{|c|c|c|}
\hline $\begin{array}{c}\text { No. of active } \\
\text { sensor nodes }\end{array}$ & $\begin{array}{c}\text { Tracking Ability Rate for } \\
\text { Face-track scheme (Existing } \\
\text { system) }\end{array}$ & $\begin{array}{c}\text { Tracking Ability Rate } \\
\text { for A-star scheme } \\
\text { (Proposed system) }\end{array}$ \\
\hline 3 & 0.602 & 2.551 \\
\hline 7 & 2.004 & 5.952 \\
\hline 11 & 3.144 & 9.345 \\
\hline 15 & 4.310 & 12.820 \\
\hline
\end{tabular}

Table 4 shows the tracking ability rate values for the Facetrack scheme and A-star scheme for target tracking.

Figure 5 shows the Tracking ability rate of Face track and A star. It can be seen that the Tracking ability Rate of the A-star is larger as compared to the Face-track scheme.

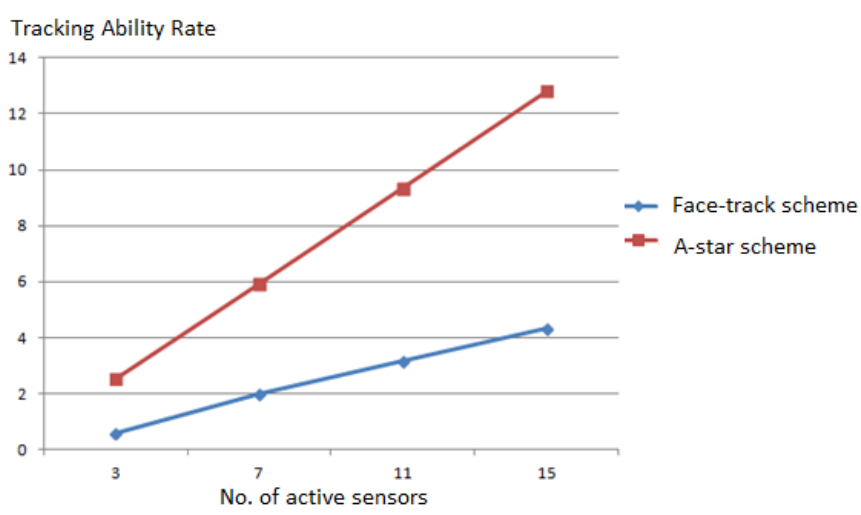

Figure 5: Tracking Ability Rate for Face-track and A- star

\section{Conclusion}

This paper proposes a polygon based target-tracking framework with Edge detection algorithm and A-star algorithm. Edge detection algorithm divides the WSN into polygon framework and makes the nodes of current polygon active while tracking and rest in the sleep mode. A-star algorithm chooses only optimal nodes that should participate in tracking process based on their average remaining energy. Proposed scheme can track the multiple targets at a time with less energy consumption and higher tracking accuracy than existing schemes.

\section{Acknowledgment}

I would like to thank my guide Prof. S. A. Nagtilak for her valuable feedback, encouragement and guidance throughout the duration of the paper. Her suggestions were of immense help throughout this paper.

\section{References}

[1] Guojun Wang, Md Zakirul Alam Bhuiyan, Jiannong Cao and Jie Wu, "Detecting Movements of a Target Using Face Tracking in Wireless Sensor Networks," IEEE Transactions on Parallel and Distributed Systems, vol. 25, no. 4, April 2014

[2] Jianxun Li and Yan Zhou, "Target Tracking in Wireless Sensor Networks," Wireless Sensor Networks: 
Application Centric Design, Yen Kheng Tan (Ed.), ISBN: 978-953-307-321-7

[3] Mohsin Fayyaz, "Classification of Object Tracking Techniques in Wireless Sensor Networks," Scientific Research, April 2011

[4] Yingqi Xu Julian Winter Wang-Chien Lee "Predictionbased Strategies for Energy Saving in Object Tracking Sensor Networks," Proceedings of the 2004 IEEE International Conference on Mobile Data Management (MDM'04)

[5] George K. Atia, Venugopal V. Veeravalli and Jason A. Fuemmeler "Sensor Scheduling for Energy-Efficient Target Tracking in Sensor Networks," IEEE Transactions on Signal Processing, Vol. 59, No. 10, October 2011

[6] Bo Jiang, Binoy Ravindran and Hyeonjoong Cho "Probability-Based Prediction and Sleep Scheduling for Energy-Efficient Target Tracking in Sensor Networks," IEEE Transactions on Mobile Computing, Vol. 12, No. 4, April 2013

[7] L.M. Kaplan, "Global Node Selection for Localization in a Distributed Sensor Network," IEEE Transactions on Aerospace and Electronic Systems, vol. 42, no. 1, pp. 113-135, January 2006

[8] L.M. Kaplan, "Local Node Selection for Localization in a Distributed Sensor Network," IEEE Transactions on Aerospace and Electronic Systems, vol. 42, no. 1, pp. 136-146, January 2006

[9] W. Zhang and G. Cao, "Dynamic Convoy Tree-Based Collaboration for Target Tracking in Sensor Networks," IEEE Transactions Wireless Communication, vol. 3, no. 5, September 2004

[10] M. Waelchli, M. Scheidegger, and T. Braun, "Intensity Based Event Localization in Wireless Sensor Networks," Proceedings Conference International Federation for Information Processing Wireless on Demand Network Systems and Services (IFIP WONS), pp. 41-49, 2006

[11] O. Kaltiokallio, M. Bocca, and L.M. Eriksson, "Distributed RSSI Processing for Intrusion Detection in Indoor Environments," Proceedings Ninth ACM IEEE International Conference Information Processing in Sensor Networks (IPSN), pp. 404-405, 2010

[12] Sandy Mahfouz, Farah Mourad-Chehade, Paul Honeine, Joumana Farah and Hichem Snoussi "Target Tracking Using Machine Learning and Kalman Filter in Wireless Sensor Networks," IEEE Sensors Journal, Vol. 14, No. 10, October 2014

[13] Ye Zhu, Anil Vikram, Huirong Fu and Yong Guan "On Non-Cooperative Multiple-Target Tracking With Wireless Sensor Networks," IEEE Transactions on Wireless Communications, Vol. 13, No. 11, November 2014

[14] A. A. Pawar, and Prof. S. A. Nagtilak, "Energy Efficient Face Tracking Scheme for Target Movement Detection in WSN", in iPGCON 2015 at Amrutvahini College of Enegineering, Sangamner.

[15]Hua-Wen Tsai, Chih-Ping Chu, Tzung-Shi Chen, "Mobile Object Tracking in Wireless Sensor Networks," 